\title{
Development of interactive Game Based on User experience Game Engine
}

\author{
Fan Kang \\ Wuhan Polytechnic University, Wuhan, China \\ Corresponding E-mail:Kangfaer22@126.com
}

Keywords: Android games, Multiplatform games, interactive games, Unity 3D games

\begin{abstract}
This paper aims to design and develop interactive game based on Unity3D engine that is a User experience game engine. The functions that Unity3D supports autonomously are very abundant. All game developments are possible such as shader, physics engine, network, terrain manipulation, audio, video, and animation, and it is enabled so that it is possible to revise, meeting demand of user according to the need. For the efficiency of game development process, this study aims to plan, design, and develop a interactive game based on User experience game engine.
\end{abstract}

\section{Introduction}

Unity3D game engine is an integrated development tool for producing other interactive contents such as video game, architectural visualization, real -time 3D animation. Its editor runs on Window, Mac OS X, so it could make games as the platforms of Window, Mac, Wii, iPad, and iPhone. It could also produce web browser game that uses unity web player plug-in. This is a similar form of flash, and it is designed so that flash user could easily adapt even with cross domain security policy and scripting [1].

IMGUI (immediate mode GUI) was introduced for realizing GUI. It has an advantage that it could realize GUI with simple and small amount of script through this, but it is difficult to write GUI in complex form and event handling procedure. Currently, the version 3.1 does not support the function of writing GUI in editor and not in script.

The functions that Unity3D supports autonomously are very abundant. In fact, all game developments are possible such as shader, physics engine, network, terrain manipulation, audio, video, and animation, and it considered so that the revision is possible to the taste of user according to the need. Unity3D that produces based on Java script and C\# can apply and manage after producing the desired functions with script, not producing all of the programing at once. GUI composed on screen helps the first-time developer to approach easily, and the script and program that programmer made with simple mouse drag [2].

This study aims to design and develop interactive game based on Unity3D engine that is a User experience game engine. The functions that Unity3D supports autonomously are very abundant. All game developments are possible such as shader, physics engine, network, terrain manipulation, audio, video, and animation, and it is enabled so that it is possible to revise, meeting the demand of user according to the need. For the efficiency of game development process, this study aims to plan, design, and develop a interactive game based on User experience game engine [3].

\section{Unity3D Game Engine}

In order to produce multiplatform games, for each platform, all the different settings of the system must be controllable. In this paper, the use of Unity3D engine is proposed based on the reason that codes can be written with C\#, JavaScript and Boo, the Unity3D engine was developed as a C\#- and Mono-based code. The run-time part of the engine was developed with $\mathrm{C}++$ and Microsoft NET API, and the editor program was developed with C\#. As for the script, it cannot be modified directly in Unity, and it can be modified in script editors, such as Mono Develop, etc., that support Unity. Although Visual Studio also supports through plug -ins, it lacks overall debugging functionality and 
in the Express version, debugging is not supported. In the case of Unity3, not only simple physics engines or shaders, but also realistically, all conditions, including network or terrain manipulation, audio, video, animations, etc., are supported. By converting web-based games and contents developed using Unity 3D into 'IOS' or 'ANDROID,' they can be serviced as interactivephone-use (Shown in Figure 1) [4-5].

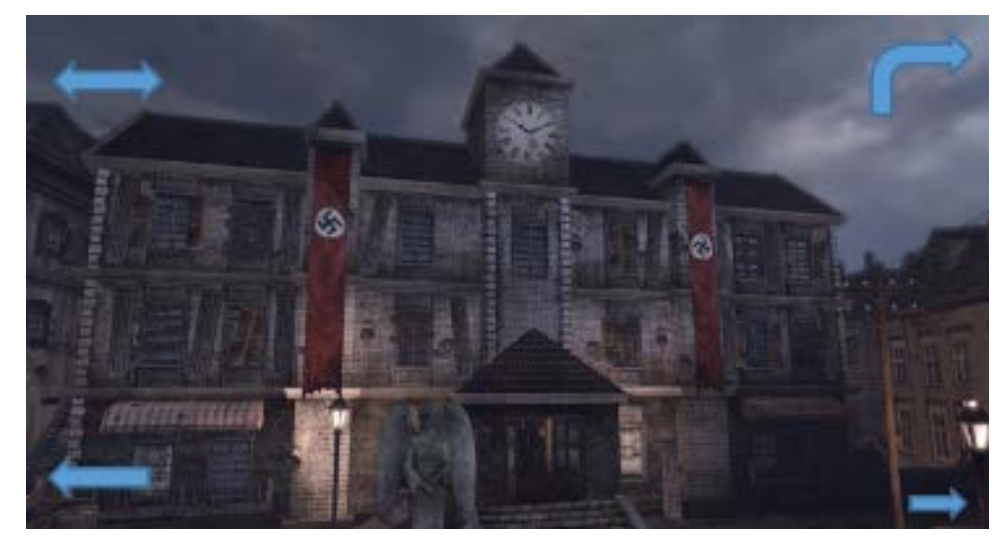

Figure 1. Unity3D Game Engine

Unity3Dsupports 3 type of script languages: Java Script, C\# and Boo. All three are capable of the same fast interaction, capable of utilizing .NET libraries, and all three support databases, standard expressions, XML, networking, etc. Even though scripting is normally considered to be limited and slow, Unity3 operates with very fast iteration times and, by pairing up with a compilation of easy native code, becomes almost as fast as the $\mathrm{C}++$ level. In addition, the implementation of Java Script for Unity3D operates at the same speed as C\# or Boo. Game logic is executed in the .NET platform Mono open source; which provides a best-in-the-world programming environment that offers the best speed and flexibility [6-7].

Application using a interactive Camera-Device has been remained on the level of recognition of business card, bar code and searching for words. The developments of game contents with upgraded technology will contribute to the growth of the application market. Therefore in this dissertation, we developed interactive augmentation reality technology introducing recognition of a marker, 3-D characters and character animation utilizing Camera-Devices based on Android platforms in order to develop health educational games for elementary students.

The platform of the game is android interactive tablet base, and the genre is sound action game. It is designed with the Battle of Thermopylae in Greece Sparta as the material.

Scenario: It is the very first menu that begins the game, and when beginning, it shows the story and instruction for how to play the game. User can select the difficulty of gameplay (Easy, Normal, and Hard) and play the game.

Custom: It is a mode that enables user to enjoy different mode other than the scenario mode (Defense, Push, Rush, and Marathon), and user can enjoy diverse event games (currently, yet to be materialized).

Shop: User can purchase items that can be helpful to gameplay. Currently, only consumable items are materialized.

Option: This is the place where user sets up the options, and user can set touch sensitivity and sound and choose whether to skip the story when the scenario is playing.

Exit: Confirm whether to end the game or not.

\section{Game Play}

Currently, only the scenario mode is materialized, and it is planned to materialize the custom mode in future. 
Before the gameplay, a short story and instruction on how to play game appear. User can move onto the next page by touching the screen, and the game continues.

Select the difficulty of scenario mode-> Scenario progress screen -> Explanation on how to continue the game-> Screen of easy gameplay of scenario-> Menu screen within the game->the screen of game ending (Victory or Defeat)

Select the difficulty of scenario mode-> Scenario progress screen -> Explanation on how to continue the game-> Screen of easy gameplay of scenario-> Menu screen within the game-> The screen of game ending (Victory or Defeat)

It shows all the explanation on the game interface, and when user plays it for the first time, it is shown at the end of the scenario. When beginning from the option window, user can check the indications.

In the window above, if user touches one more time, then it shows the instruction related to the game on the window that has appeared. Similarly, it can be set in the option window, and when touching the screen, it moves on to the beginning of the game, and the loading screen that loads the game is created in the middle.

The process is similar to the biological evolution, each a cycle will produce more excellent collection. Until the last cycle, it will produce an optimal solution. Finally perform decoding operations. The standard GA process:

(1) The initialization: number of evolutionary, crossover rate and mutation rate is initialized three parameters, and randomly generated a number of individuals.

(2) For each individual in the initial population respectively calculate the fitness of these parameters.

(3) To choose: refer to the fitness of each individual parameter; choose a scale of excellent individuals directly into the next generation.

(4) To cross reference rate for a certain a certain gene structure of the individual exchange gained excellent individuals into the next generation.

(5) To refer to mutation rates change some individuals a number of genes into the next generation.

(6) If meet the termination conditions, to get the optimum in the process of genetic offspring; Vice continue (3).

Step1. Artificial given basketball video segmentation, capture panoramic camera video similar to figure 2 period of $20 \mathrm{~s}$, and all of them as experiment frame sequence image frames;

Step2. To regional segmentation of experimental frames used way, keep the middle area.

Step3. Calculate all the frames used in HSV color components in the space the sum of the histogram $H_{\text {sum }}$.

$$
H_{\text {sum }}=\sum_{i=1}^{n} H\left(I_{i}\right)
$$

$\mathrm{n}$ is the total number of image frames in the 20s video.

Step4. Calculate the sum of all pixels Total $_{\text {sum }}$ in the $H_{\text {sum }}$.

$$
\text { Total }_{\text {sum }}=\sum_{i=1}^{k} H_{\text {sum }}
$$

$\mathrm{k}$ is for image frames color quantization level. Here we will attune (original h component of HSV space for grade 360 ) quantitative level of 40 , namely $k=40$. The experiment proved that after the quantitative stadium has good color clustering.

Step5. Calculate the average color.

$$
\overline{c o l \_s e q}=\frac{\sum_{i=1}^{n}\left(\operatorname{col}_{\text {max }_{i}}\left(\text { Total }_{\text {sum }}\right)\right)}{n}
$$




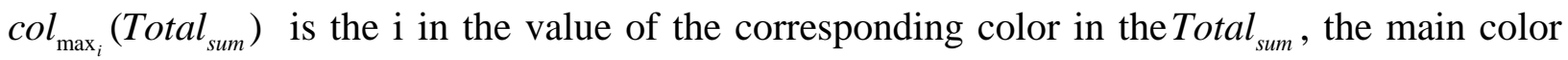
sequence number is 5 , so $n=5$, the largest of the five values corresponding to the average value in the Total $_{\text {sum }}$.Shown in fig 2.

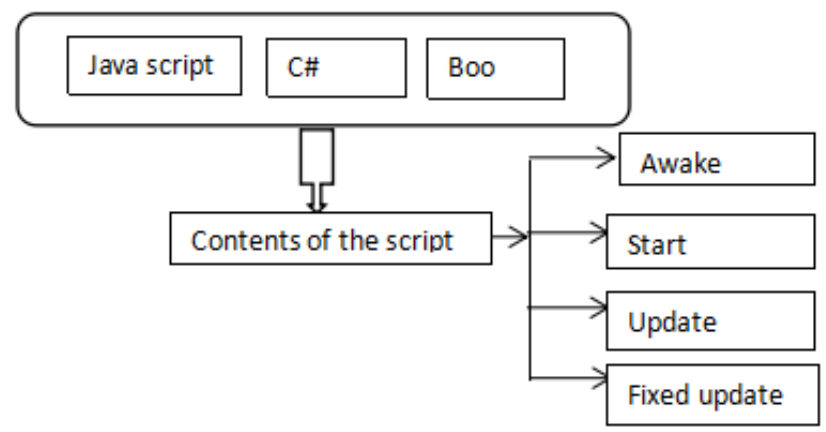

Fig 2 Flow chart

\section{Conclusion}

This study aims to design and develop a interactive game based on Unity3D engine, which is a User experience game engine. The functions that Unity3D supports autonomously are very abundant. All game developments are possible such as shader, physics engine, network, terrain manipulation, audio, video, and animation, and it is enabled so that it is possible to revise, meeting demand of user according to the need. For the efficiency of game development process, this study aims to plan, design, and develop a interactive game based on multi -platform game engine.

\section{Acknowledgment:}

This paper is funded by projects:Wuhan polytechnic university of teaching research project(XM2015021);Wuhan polytechnic university Introduced talents research initiative project (20152S05)

\section{References}

[1] J. M. Field, M. F. Hazinski and M. R. Sayre, "Part 1: executive summary: 2010 American Heart Association Guidelines for Cardiopulmonary Resuscitation and Emergency Cardiovascular Care”, Cir, (18 Suppl 3), vol. 122, (2010), pp. 640-656.

[2] M. J. Lee, “Incidence and outcome of cardiac arrest in Korea”, J Korean Social Emergency Med, vol. 23, (2012), pp. 168-180.

[3] S. O. Hwang, M. E. Ahn, Y. S. Kim, K. S. Lim, J. H. Yun and K. H. Choe, "Outcome of resuscitation victims of pre-hospital cardiac arrest”, J Korean Social Emergency Med, vol. 3, (1992), pp. 27-36.

[4] S. O. Hwang and K. S. Leem, "Cardiopulmonary Resuscitation and Advanced Cardiovascular Life Support”, Koonja publishing, Seoul, (2011).

[5] B. C. Lee, M. J. Lee, S. J. Shin, H. O. Ryu, J. K. Kim, J. B. Park and K. S. Seo, "The Current Status of Cardiopulmonary Resuscitation Training for School”, J Korean Social Emergency Med, vol. 23, no. 4, (2012), pp. 470-478.

[6] J. H. Bae and A. H. Kim, "Design and Development of Unity3D Game Engine-Based interactive SNG (Social Network Game)”, International Journal of Multimedia and Ubiquitous Engineering, Science \& Engineering Research Support Society(SERSC), vol. 8, no. 20, (2014), pp. 261-266.

[7] J. H. Bae and A. H. Kim, “A Study of the 3D Mobile User experience Game”, 2014 International Conference on Platform Technology and Service (PlatCon-14), vol. 2, no. 11, (2014), pp. 246247. 